\title{
Natural Occurrence and Distribution of Entomopathogenic Fungi from Chhattisgarh
}

\author{
Vinod Kumar Nirmalkar*, N. Lakplae* and R.K.S. Tiwari
}

Biocontrol Laboratory, BTC College of Agriculture \& Research Station, Sarkanda, Bilaspur, 495001 (I.G.K.V.) (C.G.)* Department of Plant Pathology, College of Agriculture, Raipur (I.G.K.V.) (C.G.), India

*Corresponding author

\begin{tabular}{|c|c|}
\hline & A B S T R A C T \\
\hline $\begin{array}{l}\text { Ke y w or d s } \\
\text { Entomopathogenic } \\
\text { fungi, Beauveria } \\
\text { bassiana, } \\
\text { Metarhizium } \\
\text { anisopliae, insect } \\
\text { pathogen }\end{array}$ & \multirow{3}{*}{$\begin{array}{l}\text { Extensive survey was conducted for collection of insects cadavers from } \\
\text { agriculture fields and forest area and isolated different entomopathogenic } \\
\text { fungi viz,. B. bassiana, } M \text {. anisopliae, Nomurea rileyi from different crops } \\
\text { i.e. Soybean, groundnut, Pigeonpea crop insects. The infected insects } \\
\text { cadavers was covered partially or fully with powdery white to green spores. } \\
\text { Presence of powdery spores on insects revealed B. bassiana infection, } \\
\text { green spores depicts infection of } M \text {. anisopliae or Nomuraea rileyi. } N \text {. } \\
\text { rileyi infected larvae attached to the leaves with posterior portion and } \\
\text { anterior portion of the body hanging in air and presence of yellowish spores } \\
\text { on larval body indicating Aspergillus infection. Entomopathogenic fungi } \\
\text { infected cadavers were found maximum between } 1^{\text {st }} \text { week of September to } \\
2^{\text {nd }} \text { week of January while maximum in September followed by October } \\
\text { month. }\end{array}$} \\
\hline Article Info & \\
\hline $\begin{array}{l}\text { Accepted: } \\
\text { 15 December } 2019 \\
\text { Available Online: } \\
\text { 20 January } 2020\end{array}$ & \\
\hline
\end{tabular}

\section{Introduction}

Fungi which control the insect pest population are associated with agricultural crops are called as entomopathogenic fungi. There are naturally occurring organisms, such as bacteria, viruses and fungi for the control of crop pests, which can act as a parasite of insects and kills or seriously disables them. In recent years, microbial pathogens like viruses, bacteria, fungi and protozoa have been recognized for the biological suppression of many insect pests.

About 1.5 million species of fungi alone are known to occur worldwide out of which nearly half of the species have been identified. Amongst these, several asexual stages of fungi are associated with insect 
infection. Entomopathogenic fungi are naturally occurring organisms which perceived as less damaging to the environment (Tahira, et al., 2014). Among biopesticdes, entomopathogenic fungi played a significant role in insect pathology and especially in microbial control.

Most of the entomopathogenic fungi belong to either entomopthorales (Zygomycotina) or hyphomycetes (Deuteromycotina). Prominent genera that have been exploited for the pathogenic properties include Nomuraea, Beauveria, Metarhizium, Verticillium, Hirsutella, Aspergillus, Coelomomyces, Lagenidium, Paecilomyces and Tolypocladium of which the first five are the best known cosmopolitan insect pathogens.

Occurrence and distribution of entomopathogenic fungi (EPF) in diverse habitats are divided in to main two ecosystem; manmade agriculture and natural habitat (aquatic, forest and non forest). EPF of aquatic habitats belongs to Mastigomycota and Zygomycota and these have ability to produce motile spore, presence of thick wall spore, has capacity in absence of water and adapted to semi permanent habitats. Tropical humid forests are rich in various entomopathogen and agriculture ecosystems have diverse entomopathgenic fungal species. Environmental factors i.e. temperature, humidity and light, play major role in field persistence of entomopathogenic fungi. One of the critical factors in the effective use of microbial agents is their relatively short persistence on leaf surfaces (Khachatourians, 1996).

Agro ecological condition of Chhattisgarh favored the local isolates of entomopathogenic fungi which play a vital role in suppressing pest population because their degree of virulence, survival, adoption, commercialization and successful are most importance that's why need to identify novel new indigenous isolates, shelf life, development of formulation, evaluate the efficacy of entomopathogenic fungi for cost economic, safe production, physicals factors, nutritional requirements. Very limited studies on these aspects in the state and to identified alternate biological insect management measures under organic farming in the state.

\section{Materials and Methods}

Extensive surveys were conducted during Kharif and Rabi seasons of 2017-18, 2018-19 and Kharif 2019-20 for collection of entomopathogenic fungi from different localities of Chhattisgarh state i.e. Bilaspur, Raipur, Bhatapara, Mungeli, Kawardha, Bemetara, Jangir-champa, Jagdalpur and Korba districts from farmers fields and forests of Chhattisagrh.

Thirty insect cadavers infected with fungus were collected and placed in separate sterilized glass vessels. After collection, these insect cadavers were brought to laboratory, made fairly dry to avoid further deterioration and stored in refrigerator. The collected insect cadavers were coded. During the survey different information was gathered i.e. Latitude and Longitude, crops, insects, location, seasons etc.

\section{Results and Discussion}

The extensive surveys was conducted for collection of insect cadavers from agriculture fields and forest area during Kharif and Rabi season of 2017-18, 2018-19 and Kharif of 2019 to know the occurrence, distribution and biodiversity study of indigenous fungi prevalent in Chhattisgarh. The study was conducted in different districts of Chhattisgarh state i.e. Bilaspur, Jangir champa, Mungeli, Kawardha, Bemetara, Raipur, Korba, Ambikapur and Jagdalpur 
between August and February of every year. During twenty-eight surveys, thirty insects cadavers was collected from different crops i.e. Soybean, Groundnut, Sugarcane, Paddy, Pigeonpea and Mustard of various cropping systems . Out of thirty cadavers collect 23 were from Spodoptera litura of different crops (Soybean 15; Groundnut 7 and Potato (1). Helicoverpa armigera from Pigeonpea (2), Pyrilla perpusilla from Sugarcane (1), Lipaphis erysimi from Mustard (3) and Scirpophaga incertulas from Paddy (1).

Different instars of insects were found infected with fungi showing various symptoms/colours. The infected insect's cadaver was covered partially or fully with powdery white to green spores. Powdery spores suspected with $B$. bassiana infection and infected larvae generally found in upper leaf surface. Green spores infection was mostly with $M$. anisopliae or Nomuraea rileyi. $N$. rileyi infected larvae attached to the leaves with posterior portion and anterior portion of the body hanging in air and presence of yellowish spores on larval body indicating Aspergillus infection. Entomopathogenic fungi (EPF) infected insects were generally hardened (Table 1, plate $1.1,1.2$ and 1.3).

Entomopathogenic fungi infected cadavers were found maximum between $1^{\text {st }}$ week of September to $2^{\text {nd }}$ week of January while maximum in September followed by October month. During $1^{\text {st }}$ week of September to $2^{\text {nd }}$ week of October the environmental conditions were found favourable for occurrence of insects cadavers infected with EPF in soybean and Groundnut crops. whereas $2^{\text {nd }}$ week of September to last week of December, environmental conditions favours Sugarcane crop insect infection by EPF. November to December was ideal months for infecting $H$. armigera of Pigeonpea crop. No insect cadaver was found from forest area, all were collected from agriculture habitats. It was observed that population of EPF was higher in unmanaged field.

Table.1 Details of survey for collection of entompathogenic fungi

\begin{tabular}{|l|l|l|l|l|l|l|l|}
\hline S.N. & $\begin{array}{c}\text { Season } \\
\text { IMonths of } \\
\text { Survey }\end{array}$ & Location of Survey & $\begin{array}{c}\text { Latitude and } \\
\text { longitude }\end{array}$ & Districts & \multicolumn{1}{|c|}{ Crop } & $\begin{array}{c}\text { Insect } \\
\text { Cadavers }\end{array}$ & $\begin{array}{l}\text { Sample } \\
\text { Code }\end{array}$ \\
\hline $\mathbf{1}$ & $\begin{array}{l}\text { Kharif-17/ } \\
\text { Sept }\end{array}$ & $\begin{array}{l}\text { Bilaspur- } \\
\text { BTCCARS farm }\end{array}$ & $\begin{array}{l}22^{\circ} 06^{\prime} 19.2^{\prime \prime} \mathrm{N} \\
82^{\circ} 08^{\prime} 17.2^{\prime \prime} \mathrm{E}\end{array}$ & Bilaspur & Groundnut & $\begin{array}{l}\text { Spodoptera } \\
\text { litura }\end{array}$ & EPF-01 \\
\hline $\mathbf{2}$ & $\begin{array}{l}\text { Kharif-17/ } \\
\text { Sept }\end{array}$ & $\begin{array}{l}\text { Bilaspur- } \\
\text { BTCCARS farm }\end{array}$ & $\begin{array}{l}22^{\circ} 06^{\prime} 19.4^{\prime \prime} \mathrm{N} \\
82^{\circ} 08^{\prime} 16.9^{\prime \prime} \mathrm{E}\end{array}$ & Bilaspur & Groundnut & $\begin{array}{l}\text { Spodoptera } \\
\text { litura }\end{array}$ & EPF-02 \\
\hline $\mathbf{3}$ & $\begin{array}{l}\text { Kharif-17/ } \\
\text { Sept }\end{array}$ & $\begin{array}{l}\text { Bilaspur-Farmers } \\
\text { field/Ranigaon }\end{array}$ & $\begin{array}{l}22^{\circ} 14^{\prime} 40.5^{\prime \prime} \mathrm{N} \\
82^{\circ} 08^{\prime} 23.2^{\prime \prime} \mathrm{E}\end{array}$ & Bilaspur & Sugarcane & Pyrilla & EPF-03 \\
\hline $\mathbf{4}$ & $\begin{array}{l}\text { Kharif-17/ } \\
\text { Sept }\end{array}$ & $\begin{array}{l}\text { Bilaspur- } \\
\text { BTCCARS farm }\end{array}$ & $\begin{array}{l}22^{\circ} 06^{\prime} 21.1^{\prime \prime} \mathrm{N} \\
82^{\circ} 08^{\prime} 32.9^{\prime \prime} \mathrm{E}\end{array}$ & Bilaspur & Soybean & $\begin{array}{l}\text { Spodoptera } \\
\text { litura }\end{array}$ & EPF-04 \\
\hline $\mathbf{5}$ & Kharif-17/ & Mungeli-Farmers & $22^{\circ} 03^{\prime} 37.4^{\prime \prime} \mathrm{N}$ & Mungeli & Soybean & Spodoptera & EPF-05 \\
\hline
\end{tabular}




\begin{tabular}{|c|c|c|c|c|c|c|c|}
\hline & Sept & field /Damapur & $81^{\circ} 38^{\prime} 25.3^{\prime \prime E}$ & & & litura & \\
\hline 6 & $\begin{array}{l}\text { Kharif-17/ } \\
\text { Sept }\end{array}$ & $\begin{array}{l}\text { Mungeli- Farmers } \\
\text { field /Damapur }\end{array}$ & $\begin{array}{l}22^{\circ} 03^{\prime} 35.5^{\prime \prime} \mathrm{N} \\
81^{\circ} 38^{\prime} 23.1^{\prime \prime} \mathrm{E}\end{array}$ & Mungeli & Soybean & $\begin{array}{l}\text { Spodoptera } \\
\text { litura }\end{array}$ & EPF-06 \\
\hline 7 & $\begin{array}{l}\text { Kharif-17/ } \\
\text { Sept }\end{array}$ & $\begin{array}{l}\text { Mungeli- Farmers } \\
\text { field /Chalan }\end{array}$ & $\begin{array}{l}22^{\circ} 03^{\prime} 54.3^{\prime \prime} \mathrm{N} \\
81^{\circ} 38^{\prime} 30.1^{\prime \prime} \mathrm{E}\end{array}$ & Mungeli & Soybean & $\begin{array}{l}\text { Spodoptera } \\
\text { litura }\end{array}$ & EPF-07 \\
\hline 8 & $\begin{array}{l}\text { Kharif-17/ } \\
\text { Sept }\end{array}$ & Raipur IGKV farm & $\begin{array}{l}21^{\circ} 13^{\prime} 50.8^{\prime \prime} \mathrm{N} \\
81^{\circ} 43^{\prime} 03.6^{\prime \prime} \mathrm{E}\end{array}$ & Raipur & Soybean & $\begin{array}{l}\text { Spodoptera } \\
\text { litura }\end{array}$ & EPF-08 \\
\hline 9 & $\begin{array}{l}\text { Kharif-17/ } \\
\text { Sept }\end{array}$ & Raipur IGKV farm & $\begin{array}{l}21^{\circ} 13^{\prime} 47.7^{\prime \prime} \mathrm{N} \\
81^{\circ} 43^{\prime} 01.1^{\prime \prime} \mathrm{E}\end{array}$ & Raipur & Soybean & $\begin{array}{l}\text { Spodoptera } \\
\text { litura }\end{array}$ & EPF-09 \\
\hline 10 & $\begin{array}{l}\text { Kharif-17/ } \\
\text { Sept }\end{array}$ & $\begin{array}{l}\text { Bilaspur/farmers } \\
\text { field/ Pendarwa }\end{array}$ & $\begin{array}{l}22^{\circ} 13^{\prime} 47.1^{\prime \prime N} \\
82^{\circ} 08^{\prime} 39.3^{\prime \prime} \mathrm{E}\end{array}$ & Bilaspur & Paddy & $\begin{array}{l}\text { Scirpophaga } \\
\text { incertulas }\end{array}$ & EPF-10 \\
\hline 11 & $\begin{array}{l}\text { Kharif-17/ } \\
\text { Sept }\end{array}$ & $\begin{array}{l}\text { Bhatapara/Khapara } \\
\text { dih farm } \\
\text { (DKCARS) }\end{array}$ & $\begin{array}{l}21^{\circ} 44^{\prime} 15.4^{\prime \prime} \mathrm{N} \\
81^{\circ} 58^{\prime} 16.2^{\prime \prime} \mathrm{E}\end{array}$ & Bhatapara & Soybean & $\begin{array}{l}\text { Spodoptera } \\
\text { litura }\end{array}$ & EPF-11 \\
\hline 12 & $\begin{array}{l}\text { Kharif-17/ } \\
\text { Sept }\end{array}$ & $\begin{array}{l}\text { Bhatapara/Alesure } \\
\text { farm (DKCARS) }\end{array}$ & $\begin{array}{l}21^{\circ} 43^{\prime} 46.3^{\prime \prime} \mathrm{N} \\
81^{\circ} 59^{\prime} 14.4^{\prime \prime} \mathrm{E}\end{array}$ & Bhatapara & Soybean & $\begin{array}{l}\text { Spodoptera } \\
\text { litura }\end{array}$ & EPF-12 \\
\hline 13 & $\begin{array}{l}\text { Kharif-17/ } \\
\text { Sept }\end{array}$ & $\begin{array}{l}\text { Bhatapara/ } \\
\text { Khaparadih farm } \\
\text { (DKCARS) }\end{array}$ & $\begin{array}{l}21^{\circ} 44^{\prime} 15.7^{\prime \prime N} \\
81^{\circ} 58^{\prime} 16.7^{\prime \prime} \mathrm{E}\end{array}$ & Bhatapara & Soybean & $\begin{array}{l}\text { Spodoptera } \\
\text { litura }\end{array}$ & EPF-13 \\
\hline 14 & $\begin{array}{l}\text { Kharif-17/ } \\
\text { Oct }\end{array}$ & $\begin{array}{l}\text { Kawardha/KVK } \\
\text { farm }\end{array}$ & $\begin{array}{l}22^{\circ} 01^{\prime} 37.1^{\prime \prime} \mathrm{N} \\
81^{\circ} 15^{\prime} 13.1^{\prime \prime} \mathrm{E}\end{array}$ & Kawadha & Soybean & $\begin{array}{l}\text { Spodoptera } \\
\text { litura }\end{array}$ & EPF-14 \\
\hline 15 & $\begin{array}{l}\text { Kharif-17/ } \\
\text { Oct }\end{array}$ & $\begin{array}{l}\text { Kawardha CARS } \\
\text { farm }\end{array}$ & $\begin{array}{l}21^{\circ} 59^{\prime} 14.6^{\prime \prime} \mathrm{N} \\
81^{\circ} 14^{\prime} 17.9^{\prime \prime} \mathrm{E}\end{array}$ & Kawardha & Soybean & $\begin{array}{l}\text { Spodoptera } \\
\text { litura }\end{array}$ & EPF-15 \\
\hline 16 & $\begin{array}{l}\text { Kharif-17/ } \\
\text { Oct }\end{array}$ & $\begin{array}{l}\text { Kawardha/ Farmer } \\
\text { field /Newari }\end{array}$ & $\begin{array}{l}22^{\circ} 01^{\prime} 26.5^{\prime \prime} \mathrm{N} \\
81^{\circ} 15^{\prime} 25.8^{\prime \prime} \mathrm{E}\end{array}$ & Kawardha & Soybean & $\begin{array}{l}\text { Spodoptera } \\
\text { litura }\end{array}$ & EPF-16 \\
\hline 17 & $\begin{array}{l}\text { Kharif-17/ } \\
\text { Dec }\end{array}$ & $\begin{array}{l}\text { Bilaspur/ Farmer } \\
\text { field /Amane }\end{array}$ & $\begin{array}{l}22^{\circ} 16^{\prime} 23.1 " \mathrm{~N} \\
82^{\circ} 00^{\prime} 40.1 " \mathrm{E}\end{array}$ & Bilaspur & Piegonpea & $\begin{array}{l}\text { Helicoverpa } \\
\text { armigera }\end{array}$ & EPF-17 \\
\hline 18 & $\begin{array}{l}\text { Rabi-17- } \\
\text { 18/Jan }\end{array}$ & $\begin{array}{l}\text { Bilaspur/Farmers } \\
\text { field /Amane }\end{array}$ & $\begin{array}{l}22^{\circ} 16^{\prime} 22.9^{\prime \prime} \mathrm{N} \\
82^{\circ} 00^{\prime} 38.8^{\prime \prime} \mathrm{E}\end{array}$ & Bilaspur & Mustard & $\begin{array}{l}\text { Lipaphis } \\
\text { erysimi }\end{array}$ & EPF-18 \\
\hline 19 & Rabi-17/- & Bilaspur/Farmers & $22^{\circ} 16^{\prime} 25.2^{\prime \prime} \mathrm{N}$ & Bilaspur & Mustard & Lipaphis & EPF-19 \\
\hline
\end{tabular}




\begin{tabular}{|c|c|c|c|c|c|c|c|}
\hline & $18 / \mathrm{Jan}$ & field /Amane & $82^{\circ} 00^{\prime} 39.9^{\prime \prime} \mathrm{E}$ & & & erysimi & \\
\hline 20 & $\begin{array}{l}\text { Kharif- } \\
\text { 18/August }\end{array}$ & Raipur/IGKV farm & $\begin{array}{l}21^{\circ} 13^{\prime} 47.0^{\prime \prime} \mathrm{N} \\
81^{\circ} 42^{\prime} 57.6^{\prime \prime} \mathrm{E}\end{array}$ & Raipur & Soybean & $\begin{array}{l}\text { Spodoptera } \\
\text { lituar }\end{array}$ & EPF-20 \\
\hline 21 & $\begin{array}{l}\text { Kharif } \\
18 / \text { Sept }\end{array}$ & Mungeli/Chatarkhar & $\begin{array}{l}22^{\circ} 04^{\prime} 05.1^{\prime \prime N} \\
81^{\circ} 38^{\prime} 31.5^{\prime \prime} \mathrm{E}\end{array}$ & Mungeli & Groundnut & $\begin{array}{l}\text { Spodoptera } \\
\text { litura }\end{array}$ & EPF-21 \\
\hline 22 & $\begin{array}{l}\text { Kharif } \quad- \\
\text { 18/Sept }\end{array}$ & Mungeli/Chatarkhar & $\begin{array}{l}22^{\circ} 04^{\prime} 05.3^{\prime \prime} \mathrm{N} \\
81^{\circ} 38^{\prime} 30.7 " \mathrm{E}\end{array}$ & Mungeli & Groundnut & $\begin{array}{l}\text { Spodoptera } \\
\text { litura }\end{array}$ & EPF-22 \\
\hline 23 & $\begin{array}{l}\text { Kharif } \\
18 / \text { Sept }\end{array}$ & Bilaspur/Takhatpur & $\begin{array}{l}22^{\circ} 07^{\prime} 42.4^{\prime \prime} \mathrm{N} \\
82^{\circ} 05^{\prime} 10.9^{\prime \prime} \mathrm{E}\end{array}$ & Bilaspur & Groundnut & $\begin{array}{l}\text { Spodoptera } \\
\text { litura }\end{array}$ & EPF-23 \\
\hline 24 & $\begin{array}{l}\text { Kharif } \\
18 / \text { Sept }\end{array}$ & $\begin{array}{l}\text { Bhatapara/Sendri } \\
\text { farm (DKCARS) }\end{array}$ & $\begin{array}{l}21^{\circ} 44^{\prime} 16.4^{\prime \prime} \mathrm{N} \\
81^{\circ} 58^{\prime} 15.4^{\prime \prime} \mathrm{E}\end{array}$ & Bhataparar & Soybean & $\begin{array}{l}\text { Spodoptera } \\
\text { litura }\end{array}$ & EPF-24 \\
\hline 25 & $\begin{array}{l}\text { Kharif } \\
18 / \text { Sept }\end{array}$ & $\begin{array}{l}\text { Bemetara/farmers } \\
\text { field/ Chandi }\end{array}$ & $\begin{array}{l}21^{\circ} 26^{\prime} 28.5^{\prime \prime} \mathrm{N} \\
81^{\circ} 27^{\prime} 32.6^{\prime \prime} \mathrm{E}\end{array}$ & Bemetara & Soybean & $\begin{array}{l}\text { Spodoptera } \\
\text { litura }\end{array}$ & EPF-25 \\
\hline 26 & $\begin{array}{l}\text { Kharif } \\
18 / \text { Sept }\end{array}$ & $\begin{array}{l}\text { Bilaspur/BTCCAR } \\
\text { S farm }\end{array}$ & $\begin{array}{l}22^{\circ} 06^{\prime} 19.2^{\prime \prime} \mathrm{N} \\
82^{\circ} 08^{\prime} 16.8^{\prime \prime} \mathrm{E}\end{array}$ & Bilaspur & Groundnut & $\begin{array}{l}\text { Spodoptera } \\
\text { litura }\end{array}$ & EPF-26 \\
\hline 27 & $\begin{array}{l}\text { Rabi18- } \\
\text { 19/Jan }\end{array}$ & $\begin{array}{l}\text { Bilaspur/BTCCAR } \\
\text { S farm }\end{array}$ & $\begin{array}{l}22^{\circ} 06^{\prime} 28.0^{\prime \prime} \mathrm{N} 82^{\circ} 0 \\
8^{\prime} 21.7^{\prime \prime} \mathrm{E}\end{array}$ & Bilaspur & Mustard & $\begin{array}{l}\text { Lipaphis } \\
\text { erysimi }\end{array}$ & EPF-19 \\
\hline 28 & $\begin{array}{l}\text { Kharif } \\
18 / \text { Sept }\end{array}$ & $\begin{array}{l}\text { Bilaspur farmer } \\
\text { field / }\end{array}$ & $\begin{array}{l}22^{\circ} 12^{\prime} 37.1^{\prime \prime} \mathrm{N} \\
82^{\circ} 07^{\prime} 05.7^{\prime \prime} \mathrm{E}\end{array}$ & Bilaspur & Groundnut & $\begin{array}{l}\text { Spodoptera } \\
\text { litura }\end{array}$ & EPF-28 \\
\hline 29 & $\begin{array}{l}\text { Kharif- } \\
\text { 19/Sept }\end{array}$ & $\begin{array}{l}\text { Ambikapur/Mainpat } \\
\text {, KVK field }\end{array}$ & $\begin{array}{l}22^{\circ} 466^{\prime} 05.5^{\prime \prime} \mathrm{N} \\
83^{\circ} 15^{\prime} 53.3^{\prime \prime} \mathrm{E}\end{array}$ & Ambikapur & Potato & $\begin{array}{l}\text { Spodoptera } \\
\text { litura }\end{array}$ & EPF-29 \\
\hline 30 & $\begin{array}{l}\text { Kharif- } \\
\text { 19/Nov }\end{array}$ & Bilaspur KVK Farm & $\begin{array}{l}22^{\circ} 06^{\prime} 18.4^{\prime \prime} \mathrm{N} \\
82^{\circ} 08^{\prime} 41.5^{\prime \prime} \mathrm{E}\end{array}$ & Bilaspur & Pigeonpea & $\begin{array}{l}\text { Helicoverpa } \\
\text { armigera }\end{array}$ & EPF-30 \\
\hline
\end{tabular}



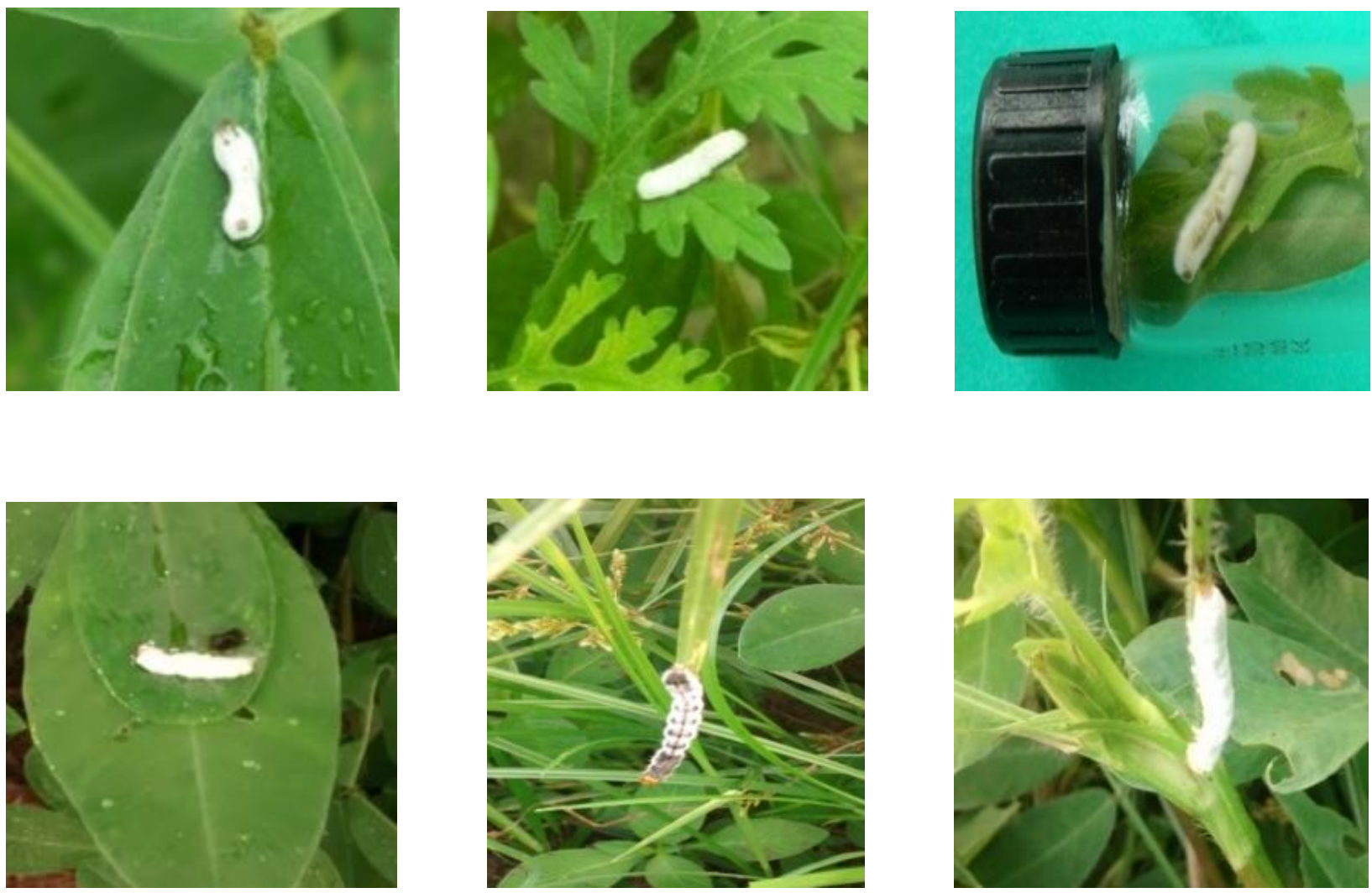

Collection of insect cadavers from Groundnut fields
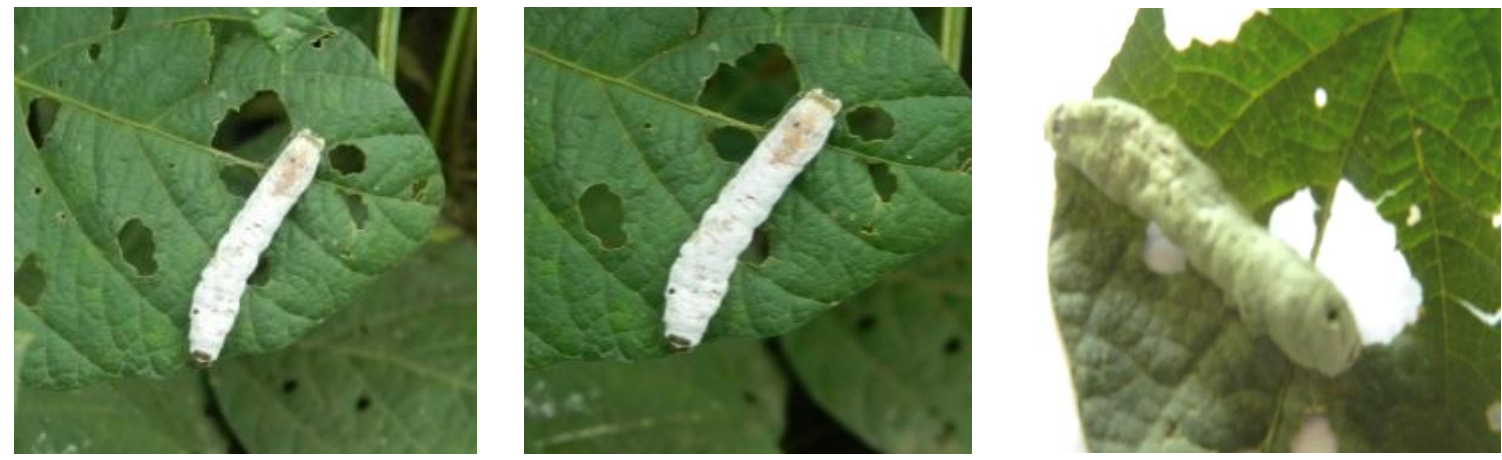

Insect cadavers from Soybean fields

Plate.1.1 Collection of insect cadavers from different location showing varying symptoms of infection of B. bassiana 

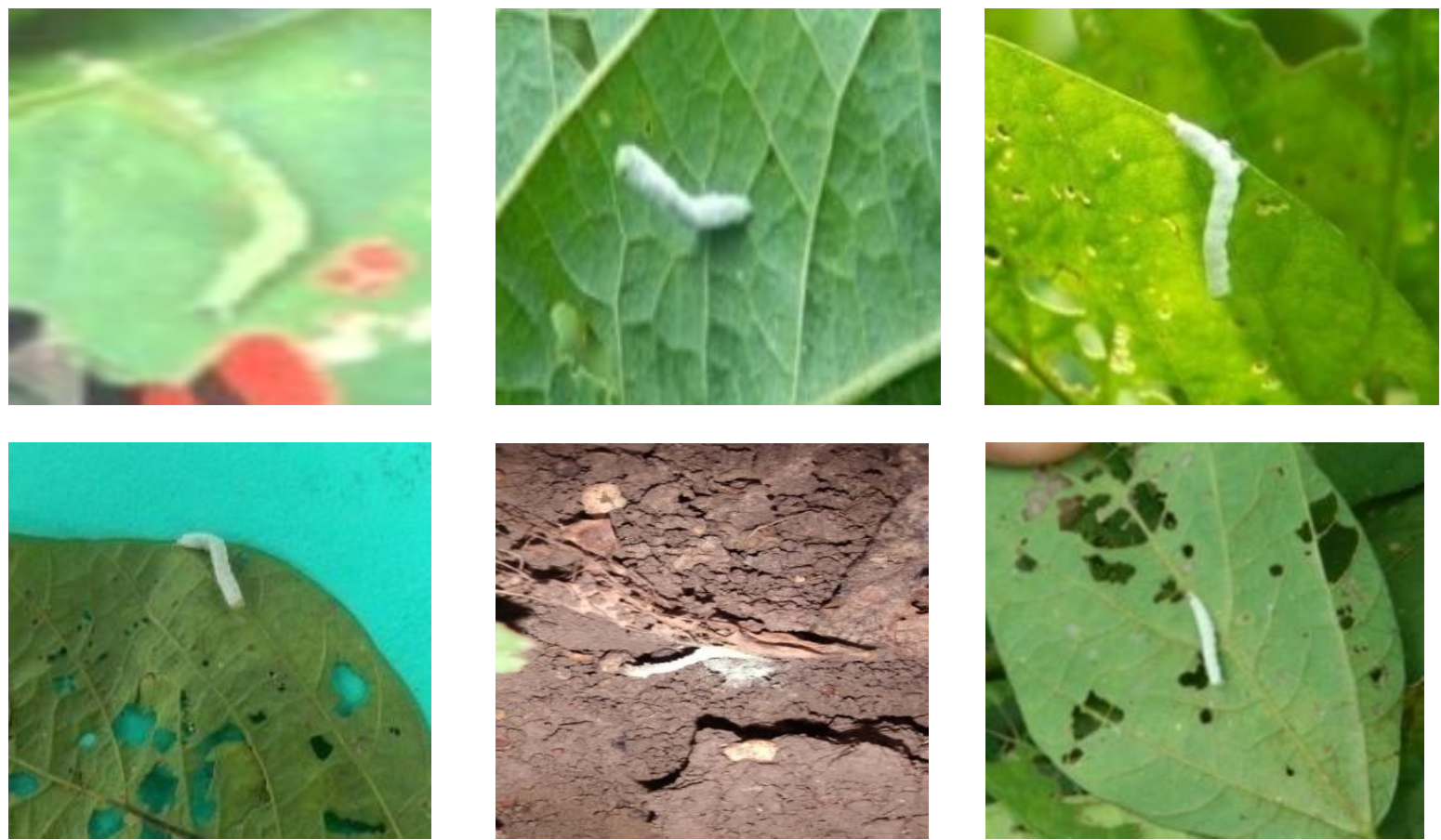

Plate 1.2 Typical symptoms of larvae infected by Nomuraea rileyi (1-6)
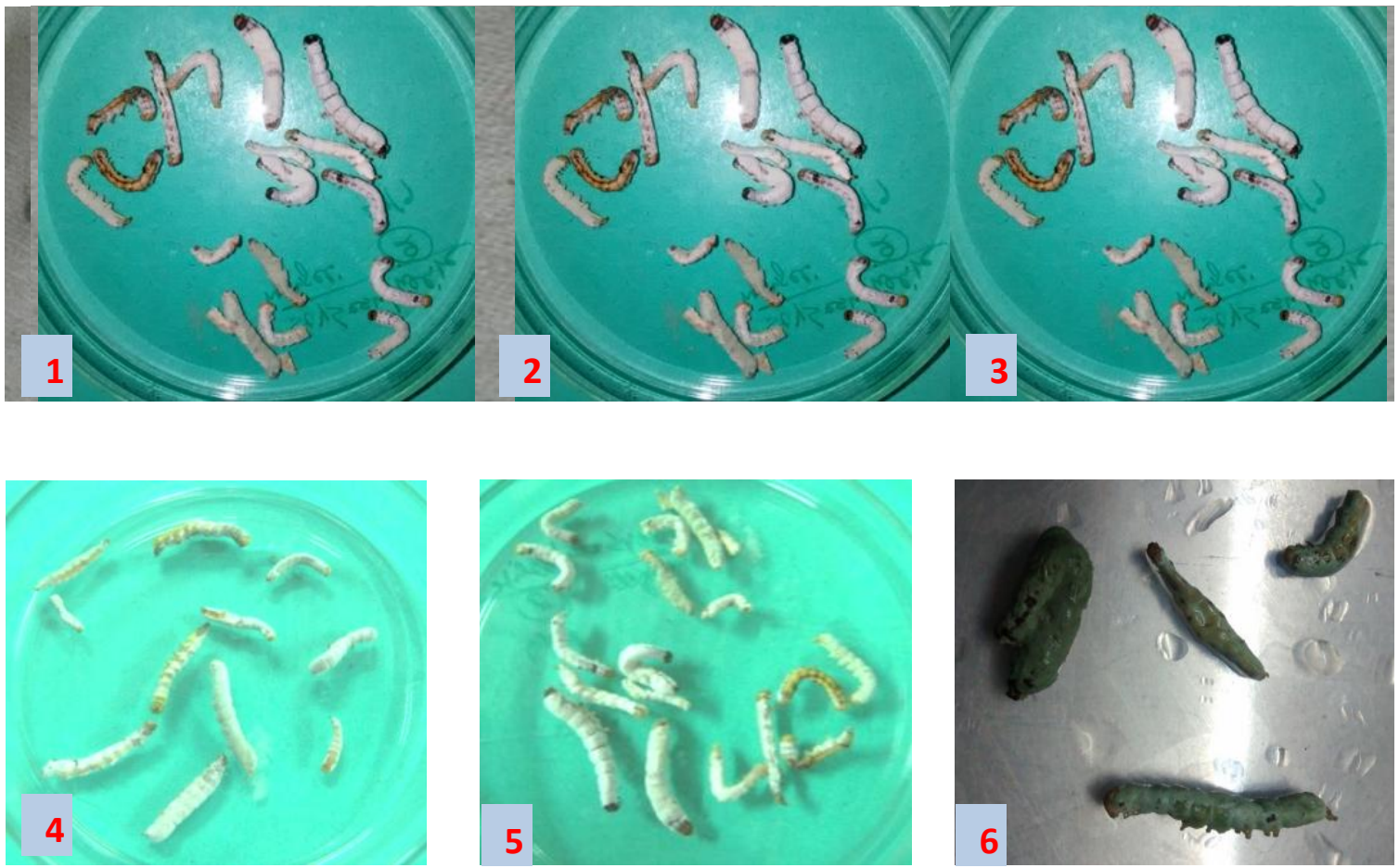

Collection of insect cadavers from different farmers field in soybean crop Plate.1.3 Different instars of larvae of Spodoptera litura (1-6) 
Survey findings indicated that for the infection of EPF on insects, moderate to low temperature along with moisture required. Proper moisture and moderate temperature were maintained naturally during the months of September to October and goes slow down. Similarly in December to January temperature goes low and proper moisture was maintained.

Various researchers were also doing such types of survey from agricultural fields, conserved and reserved forest and finding agreed to our results as they concluded that mostly EPF were collected from September to January from various orthopodos, Hemiptera, Homoptera insects. Gupta (2003) suggesed rainly days and amount of rainfall also play a role for infection of EPF, Thakur and Sandhu (2010); Prasad et al., (2011); Omoloye et al., (2015); Moorthi et al., (2015); Nidhi et al., (2018); Clifton et al., (2018) also reported that mostly cadavers were collected from soybean crop and maximum from dense crops.

\section{References}

Clifton, E.H., Jaronski, S.T., Coates, B.S., Hodgson, E.W and Gassmann, A.J. 2018. Effects of endophytic entomopathogenic fungi on soybean aphid and identification of Metarhizium isolates from agricultural fields. 13(3): 194-198.

Gupta, V. P. 2003. Natural occurrence of the entomopathogenic fungus Nomuraea rileyi in the soybean green semilooper, Chrysodeixis acuta, in India. Online. Plant Health Progress
doi:10.1094/PHP-2003-0113-01-HN. Khachatourians, G.G. 1996. Biochemistry and molecular biology of entomopathogenic fungi, In: Human and animal relationships Mycota VI (DH Howard and JD Miller, eds.). Springer, Heidelberg. 331-363.

Moorthi, V., Balasubramanian, J., Ramar, C., Murugan, K. 2015. Biocontrol Potential of Entomopathogenic Fungi against Spodoptera Litura. Scientia Agriculturae, 12 (1), 23-27.

Nidhi, K., Gaur, N and Pandey, R. 2018. A survey of the local isolates of entomopathogenic fungi in Uttarakhand region. Journal of Entomology and Zoology Studies. 6(1): 1725-1730

Omoloye, A.A., Ajifolokun, A. O. and Tobih, F.O.2015. Sources and abundance of fungi with entomopathogenic potential for control of the cowpea pod borer, Maruca vitrata Fab. in Ibadan, Nigeria. Journal of Entomology and Nematology. 7(3):18-25.

Prasad, R., Prabhu, S.T. and Balikai, R. A. 2011, Status of insect pest and their natural enemies on rice under rainfed ecosystems. Int. J. Agricult. Stat. Sci., 7(2): 473-482.

Tahira, G., Shafqat, S. and Khan, A. 2014. Entomopathogenic fungi as effective insect pest management tactic: a review. Applied Sci. and Business . I (1): 10-18.

Thakur, R. and Sandhu, S. 2010.Distribution, occurrence and natural invertebrate hosts of indigenous entomopathogenic fungi of Central India. Indian $\mathrm{J}$. Microbiol. 50(1): 89-96. 


\section{How to cite this article:}

Vinod Kumar Nirmalkar, N. Lakplae and Tiwari. R.K.S. 2020. Natural Occurrence and Distribution of Entomopathogenic Fungi from Chhattisgarh. Int.J.Curr.Microbiol.App.Sci. 9(01): 1990-1998. doi: https://doi.org/10.20546/ijcmas.2020.901.225 